\title{
STAT3 gene polymorphisms and susceptibility to non-small cell lung cancer
}

\author{
B. Jiang ${ }^{1}$, Z.Z. Zhu' ${ }^{2}$, F. Liu ${ }^{3}$, L.J. Yang ${ }^{4}$, W.Y. Zhang ${ }^{3}$, H.H. Yuan ${ }^{3}$, \\ J.G. Wang ${ }^{3}$, X.H. Hu ${ }^{3}$ and G. Huang ${ }^{1}$ \\ 'Department of Nuclear Medicine, Renji Hospital, School of Medicine, \\ Shanghai Jiaotong University, Shanghai, P.R. China \\ ${ }^{2}$ Department of Oncology, No. 113 Hospital of People's Liberation Army, \\ Ningbo, P.R. China \\ ${ }^{3}$ Department of Oncology, No. 3 People's Hospital, School of Medicine, \\ Shanghai Jiaotong University, Shanghai, P.R. China \\ ${ }^{4}$ Department of Oncology, Changhai Hospital, \\ Second Military Medical University, Shanghai, P.R. China
}

Corresponding author: G. Huang

E-mail: gang_huang@163.com

Genet. Mol. Res. 10 (3): 1856-1865 (2011)

Received September 20, 2010

Accepted May 2, 2011

Published August 26, 2011

DOI http://dx.doi.org/10.4238/vol10-3gmr1071

\begin{abstract}
Signal transducer and activator of transcription protein 3 (STAT3) has been implicated in cancer development and is recognized as a type of oncogene. However, association studies of single nucleotide polymorphisms (SNPs) in the STAT3 gene with cancer risk are rare and not available for lung cancer. We examined whether STAT3 polymorphisms are associated with the risk of non-small cell lung cancer (NSCLC). Eight SNPs in the STAT3 gene were genotyped by TaqMan assays in 326 NSCLC cases and 432 controls in a Chinese population. Significant decreased risk of NSCLC was observed for carriers of minor alleles rs4796793 (odds ratio $(\mathrm{OR})=0.68,95 \%$ confidence interval $(\mathrm{CI})=0.51-0.92)$, rs $7211777(\mathrm{OR}=$ $0.67,95 \% \mathrm{CI}=0.50-0.90), \mathrm{rs} 12949918(\mathrm{OR}=0.73,95 \% \mathrm{CI}=0.54-0.97)$, rs $744166(\mathrm{OR}=0.69,95 \% \mathrm{CI}=0.51-0.92), \mathrm{rs} 9912773(\mathrm{OR}=0.75,95 \% \mathrm{CI}=$ $0.55-0.98)$, and $\mathrm{rs} 3869550(\mathrm{OR}=0.70,95 \% \mathrm{CI}=0.53-0.94)$. The GGCGGC haplotype, comprised of minor alleles of the six NSCLC-associated SNPs,
\end{abstract}


had a 0.78 -fold $(95 \% \mathrm{CI}=0.62-0.97)$ significantly decreased risk of NSCLC, as compared to the most common haplotype of CATACT. Stratification analyses by clinical stage showed that the trend for the association between STAT3 polymorphisms and NSCLC risk was present both for stage I/II and stage III/IV, and appeared moderately stronger for stage III/IV. We conclude that polymorphisms in the STAT3 gene may have a protective role in the development of NSCLC, particular of stage III/IV NSCLC.

Key words: Non-small cell lung cancer; Polymorphisms; STAT3

\section{INTRODUCTION}

Lung cancer, predominantly non-small cell lung cancer (NSCLC), is the leading cause of cancer-related deaths worldwide (Parkin et al., 2005). In mainland China, the mortality rate for lung cancer is 41.8 for men and 19.3 for women per million individuals every year (Yang et al., 2004). Smoking has been established to be a major risk factor for lung cancer (Shields, 2002). However, not all individuals exposed to tobacco smoke develop lung cancer, suggesting that genetic susceptibility plays an important role in lung carcinogenesis (Scagliotti et al., 2009).

Signal transducer and activator of transcription protein 3 (STAT3), a member of a transcription factor family that mediates various biological responses induced by cytokines and growth factors (Turkson, 2004), is implicated in cancer development and progression and has, therefore, been recognized as a type of oncogene (Darnell, 2005; Fletcher et al., 2009; Yu et al., 2009). STAT3 participates in a series of tumorigenic cellular processes such as cell proliferation, survival, apoptosis, angiogenesis, and immune responses (Karin, 2006; Grivennikov and Karin, 2010). Constitutively activated STAT3 activity has been routinely observed in multiple human cancers including lung, gastric, skin, head and neck, ovarian, breast, colon, and prostate (Bromberg et al., 1999; Bowman et al., 2000; Yu and Jove, 2004; Hsieh et al., 2005; Lin et al., 2005; Yin et al., 2006; Sansone et al., 2007; Abdulghani et al., 2008). In vitro and animal models have shown that inactivation of STAT3 inhibited carcinogenesis and the growth of established tumors, while enhancing expression of this gene promoted tumor incidence and growth (Yu and Jove, 2004; Jing et al., 2006; Siddiquee et al., 2007a,b; Weerasinghe et al., 2007; Bollrath et al., 2009). Moreover, activation of STAT3 has been associated with advanced stages of prostate cancer (Horinaga et al., 2005) and metastatic progression of several different cancer types, including lung cancer (Dauer et al., 2005).

Despite the important role of STAT3 in cancer development and progression, association studies on single nucleotide polymorphisms (SNPs) in the STAT3 gene with risk of cancer have been rare (Vaclavicek et al., 2007) and there is no data on STAT3 polymorphisms and risk of lung cancer. It has been reported that overexpression of STAT3 is correlated with growth, survival, and radioresistance of NSCLC cells, and that STAT3 inhibition could inhibit tumorigenic ability and enhance the radiosensitivity of NSCLC cells both in vitro and in vivo (Yin et al., 2010; Hsu et al., 2011). Moreover, STAT3 polymorphisms have been shown to correlate with STAT3 expression and therapy response in certain malignancies (Ito et al., 2007; Kreil et al., 2010). Therefore, identification of STAT3 polymorphisms that are associated with NSCLC risk (either increased or decreased) is important, because it could help predict therapy response, particularly to EGFR inhibitors as STAT3 has been shown to be required 
to mediate the oncogenic effects of EGFR in NSCLC (Lai and Johnson, 2010). In the present study, we examined the association between common polymorphisms in the STAT3 gene and risk of NSCLC in a case-control study of 326 cases with NSCLC and 432 controls frequencymatched to the cases by gender, age and cigarette smoking in a Chinese population. We also examined whether the potential association of STAT3 polymorphisms with NSCLC cancer risk differs according to clinical stage status.

\section{MATERIAL AND METHODS}

\section{Subjects}

This hospital-based case-control study consisted of 326 individuals with NSCLC and 432 as cancer-free controls. All cases were prospectively recruited from No. 3 People's Hospital, Shanghai Jiaotong University, and Changhai Hospital, Second Military Medical University, Shanghai, China, between July 2005 and April 2008. They were newly diagnosed and histopathologically confirmed, without prior history of other cancers or previous chemotherapy or radiotherapy. Controls were randomly selected from non-cancer patients admitted to the same hospitals during the same period when the case patients were recruited. The control subjects were frequency-matched to the cases by gender, age ( \pm 5 years) and cigarette smoking. Clinical data were systematically recorded at entry, including age, gender, smoking history, histology type, and clinical stage. An ever-smoker was defined as a smoker of at least 1 cigarette per day for 6 months or longer. Written informed consent was obtained from each subject. This study was approved by the Ethics Review Committee of the Institutional Review Board of the two participating hospitals.

\section{SNP selection and genotyping}

The SNPs in the STAT3 gene that we examined included rs4796793, rs 7211777 , rs12949918, rs744166, rs9912773, rs3869550, rs2293152, and rs1053004, which span $76 \mathrm{~kb}$ of the STAT3 gene locus from 5'-flanking region to 3'UTR. These SNPs were selected based on i) previously published associations with various diseases (Ito et al., 2007; Vaclavicek et al., 2007; Barrett et al., 2008), thus increasing the chance of selecting SNPs with functional consequences, and ii) minor allele frequency of $\geq 0.1$ in the Chinese population, using HapMap and National Center for Biotechnology Information dbSNP public databases.

Genomic DNA was isolated from blood leukocytes by proteinase K digestion and phenol/chloroform extraction. All 8 SNPs in STAT3 were genotyped using pre-designed TaqMan SNP genotyping assays, which were ordered from Applied Biosystems (Foster City, CA, USA): C_27977213_10 (rs4796793), C_1952182_10(rs7211777), C_11628926_10 (rs12949918), C_3140282 10 (rs744166), C_11628917_10(rs9912773), C_7530575_10 (rs3869550), C_3140302_1_(rs2293152), and C_1795285_1_(rs1053004). Information on assay conditions, polymerase chain reaction (PCR) primers, and probes is available upon request (https://products.appliedbiosystems.com/ab/en/US/adirect/ab). As a lab internal quality control, four human DNA controls as well as no-template controls were run with study samples for each assay. All genotyping assays were performed on the 7900HT Fast Real-Time PCR System, and the resulting data were analyzed using the SDS 2.2 software 
(Applied Biosystems). The call rate was $\geq 99.6 \%$ for each SNP tested. Approximately $5 \%$ blind quality control samples from 2 individuals were interspersed with the study samples, achieving $\geq 99.5 \%$ concordance.

\section{Statistical analyses}

Differences in age, gender, and cigarette smoking between NSCLC cases and controls were evaluated using the $\chi^{2}$ test. Departure from Hardy-Weinberg equilibrium (HWE) among controls was assessed using the asymptotic Pearson $\chi^{2}$ test. The pair-wise linkage disequilibrium (LD) between the SNPs in STAT3 was quantified using the Haploview 4.2 software (Barrett et al., 2005). The STAT3 haplotypes were reconstructed from genotype data using the Phase 2.1 software (Stephens et al., 2001). The associations of STAT3 genotypes and haplotypes with NSCLC risk were estimated by computing the odds ratios (ORs) and 95\% confidence intervals (CIs) from multivariate logistic regression analyses with adjustment for age, gender and cigarette smoking. All tests were two-sided and a P value of less than 0.05 was considered to be significant. Statistical analyses were conducted using the Stata 10.1 software (Stata Corporation, College Station, TX, USA).

\section{RESULTS}

The general characteristics of the NSCLC cases and controls are shown in Table 1. As expected, no significant differences between cases and controls were found in the distributions of age, gender or cigarette smoking. A total of 202 adenocarcinomas, 98 squamous cell carcinomas and 26 large cell carcinomas were included in the study. The clinical stage distribution of these cases was as follows: 88 stage I, 56 stage II, 119 stage III, and 63 stage IV.

\begin{tabular}{|c|c|c|c|}
\hline Variable & Controls $(\mathrm{N}=432)$ & Cases $(\mathrm{N}=326)$ & $\mathrm{P}$ \\
\hline \multicolumn{4}{|l|}{ Age (years) } \\
\hline$\leq 60$ & $223(51.6 \%)$ & $167(51.2 \%)$ & \\
\hline$>60$ & $209(48.4 \%)$ & $159(48.8 \%)$ & 0.92 \\
\hline \multicolumn{4}{|l|}{ Gender } \\
\hline Female & $140(32.4 \%)$ & $104(31.9 \%)$ & \\
\hline Male & $292(67.6 \%)$ & $222(68.1 \%)$ & 0.88 \\
\hline \multicolumn{4}{|l|}{ Cigarette smoking } \\
\hline Never & $189(43.7 \%)$ & $138(42.3 \%)$ & \\
\hline Ever & $243(56.3 \%)$ & $188(57.7 \%)$ & 0.70 \\
\hline \multicolumn{4}{|l|}{ Histology type } \\
\hline Adenocarcinoma & & $202(61.9 \%)$ & \\
\hline Squamous cell carcinoma & & $98(30.1 \%)$ & \\
\hline Large cell carcinoma & & $26(8.0 \%)$ & \\
\hline \multicolumn{4}{|l|}{ Clinical stage } \\
\hline I & & $88(27.0 \%)$ & \\
\hline II & & $56(17.2 \%)$ & \\
\hline III & & $119(36.5 \%)$ & \\
\hline IV & & $63(19.3 \%)$ & \\
\hline
\end{tabular}

Data are reported as number with percentage in parentheses.

All SNPs tested were in HWE among control subjects. As shown in Table 2, a significant decrease in NSCLC risk was observed among carriers of minor allele of 
$\mathrm{rs} 4796793(\mathrm{OR}=0.68,95 \% \mathrm{CI}=0.51-0.92), \mathrm{rs} 7211777(\mathrm{OR}=0.67,95 \% \mathrm{CI}=0.50-0.90)$, rs $12949918(\mathrm{OR}=0.73,95 \% \mathrm{CI}=0.54-0.97), \mathrm{rs} 744166(\mathrm{OR}=0.69,95 \% \mathrm{CI}=0.51-$ $0.92), \mathrm{rs} 9912773(\mathrm{OR}=0.75,95 \% \mathrm{CI}=0.55-0.98)$, and $\mathrm{rs} 3869550(\mathrm{OR}=0.70,95 \% \mathrm{CI}$ $=0.53-0.94)$, compared to their homozygote carriers of common alleles. No significant associations with NSCLC risk were observed for the remaining two SNPs, rs2293152 and rs1053004. The interactions between rs4796793, rs7211777, rs12949918, rs744166, rs9912773, or rs3869550 and NSCLC risk factors, including age, gender, and cigarette smoking, in relation to NSCLC risk, were not statistically significant (data not shown).

Table 2. Association of STAT3 SNP genotypes and haplotypes with NSCLC risk.

\begin{tabular}{|c|c|c|c|}
\hline SNP & Controls N (\%) & Cases N (\%) & $\mathrm{OR}(95 \% \mathrm{CI})^{\dagger}$ \\
\hline \multicolumn{4}{|l|}{ rs4796793 } \\
\hline $\mathrm{CC}$ & $159(36.9)$ & $150(46.0)$ & 1.00 (Reference) \\
\hline CG & 205 (47.6) & $136(41.7)$ & $0.70(0.51-0.96)$ \\
\hline GG & $67(15.5)$ & $40(12.3)$ & $0.63(0.40-0.99)$ \\
\hline $\mathrm{CG}$ or $\mathrm{GG}$ & $272(63.1)$ & $176(54.0)$ & $0.68(0.51-0.92)$ \\
\hline \multicolumn{4}{|l|}{ rs 7211777} \\
\hline AA & $158(36.7)$ & $151(46.3)$ & 1.00 (Reference) \\
\hline $\mathrm{AG}$ & $205(47.5)$ & 135 (41.4) & $0.69(0.50-0.94)$ \\
\hline GG & $68(15.8)$ & $40(12.3)$ & $0.61(0.39-0.96)$ \\
\hline $\mathrm{AG}$ or $\mathrm{GG}$ & $273(63.3)$ & $175(53.7)$ & $0.67(0.50-0.90)$ \\
\hline \multicolumn{4}{|l|}{ rs12949918 } \\
\hline TT & $161(37.5)$ & $147(45.2)$ & 1.00 (Reference) \\
\hline TC & $202(47.1)$ & $143(44.0)$ & $0.78(0.57-1.06)$ \\
\hline $\mathrm{CC}$ & $66(15.4)$ & $35(10.8)$ & $0.58(0.36-0.92)$ \\
\hline $\mathrm{TC}$ or $\mathrm{CC}$ & $268(62.5)$ & $178(54.8)$ & $0.73(0.54-0.97)$ \\
\hline \multicolumn{4}{|l|}{ rs744166 } \\
\hline AA & $160(37.0)$ & $150(46.0)$ & 1.00 (Reference) \\
\hline $\mathrm{AG}$ & $206(47.7)$ & $137(42.0)$ & $0.71(0.52-0.97)$ \\
\hline GG & $66(15.3)$ & $39(12.0)$ & $0.63(0.40-0.99)$ \\
\hline $\mathrm{AG}$ or $\mathrm{GG}$ & $272(63.0)$ & $176(54.0)$ & $0.69(0.51-0.92)$ \\
\hline \multicolumn{4}{|l|}{ rs9912773 } \\
\hline $\mathrm{CC}$ & $172(39.8)$ & $152(46.6)$ & 1.00 (Reference) \\
\hline CG & $198(45.8)$ & $136(41.7)$ & $0.78(0.57-1.05)$ \\
\hline GG & $62(14.4)$ & $38(11.7)$ & $0.67(0.42-1.07)$ \\
\hline CG or GG & $260(60.2)$ & $174(53.4)$ & $0.75(0.55-0.98)$ \\
\hline \multicolumn{4}{|l|}{ rs 3869550} \\
\hline TT & $161(37.3)$ & $149(45.7)$ & 1.00 (Reference) \\
\hline TC & $205(47.4)$ & $137(42.0)$ & $0.72(0.53-0.99)$ \\
\hline $\mathrm{CC}$ & $66(15.3)$ & $40(12.3)$ & $0.65(0.41-1.02)$ \\
\hline $\mathrm{TC}$ or $\mathrm{CC}$ & $271(62.7)$ & $177(54.3)$ & $0.70(0.53-0.94)$ \\
\hline \multicolumn{4}{|l|}{ rs 2293152} \\
\hline $\mathrm{CC}$ & $119(27.6)$ & $82(25.2)$ & 1.00 (Reference) \\
\hline $\mathrm{CG}$ & $211(48.8)$ & $165(50.8)$ & $1.14(0.81-1.62)$ \\
\hline GG & $102(23.6)$ & $78(24.0)$ & $1.11(0.74-1.68)$ \\
\hline CG or GG & $313(72.4)$ & $243(74.8)$ & $1.13(0.81-1.57)$ \\
\hline \multicolumn{4}{|l|}{ rs1053004 } \\
\hline AA & $173(40.1)$ & $148(45.4)$ & 1.00 (Reference) \\
\hline AG & $205(47.4)$ & $136(41.7)$ & $0.78(0.57-1.06)$ \\
\hline GG & $54(12.5)$ & $42(12.9)$ & $0.91(0.57-1.44)$ \\
\hline $\mathrm{AG}$ or $\mathrm{GG}$ & $259(59.9)$ & $178(54.6)$ & $0.81(0.60-1.08)$ \\
\hline \multicolumn{4}{|l|}{ Haplotype } \\
\hline CATACT & $517(59.8)$ & $424(65.0)$ & 1.00 (Reference) \\
\hline GGCGGC & $312(36.1)$ & $199(30.5)$ & $0.78(0.62-0.97)$ \\
\hline GGCAGC & $15(1.8)$ & $11(1.7)$ & $0.89(0.40-1.96)$ \\
\hline Other haplotypes ${ }^{\S}$ & $20(2.3)$ & $18(2.8)$ & $1.10(0.57-2.10)$ \\
\hline
\end{tabular}


LD analysis, based on genotype data of the control subjects, revealed that the six NSCLC-associated SNPs (rs4796793, rs7211777, rs12949918, rs744166, rs9912773, and rs3869550) were in strong LD ( $\mathrm{D}^{\prime} \geq 0.96, r^{2} \geq 0.88$ ), and formed a haplotype block in the Chinese population (Figure 1). Haplotype association analysis showed that the GGCGGC haplotype, comprised of minor alleles of the six NSCLC-associated SNPs, was associated with a significantly decreased risk of NSCLC $(\mathrm{OR}=0.78,95 \% \mathrm{CI}=0.62-0.97)$, as compared to the most common haplotype of CATACT (Table 2).

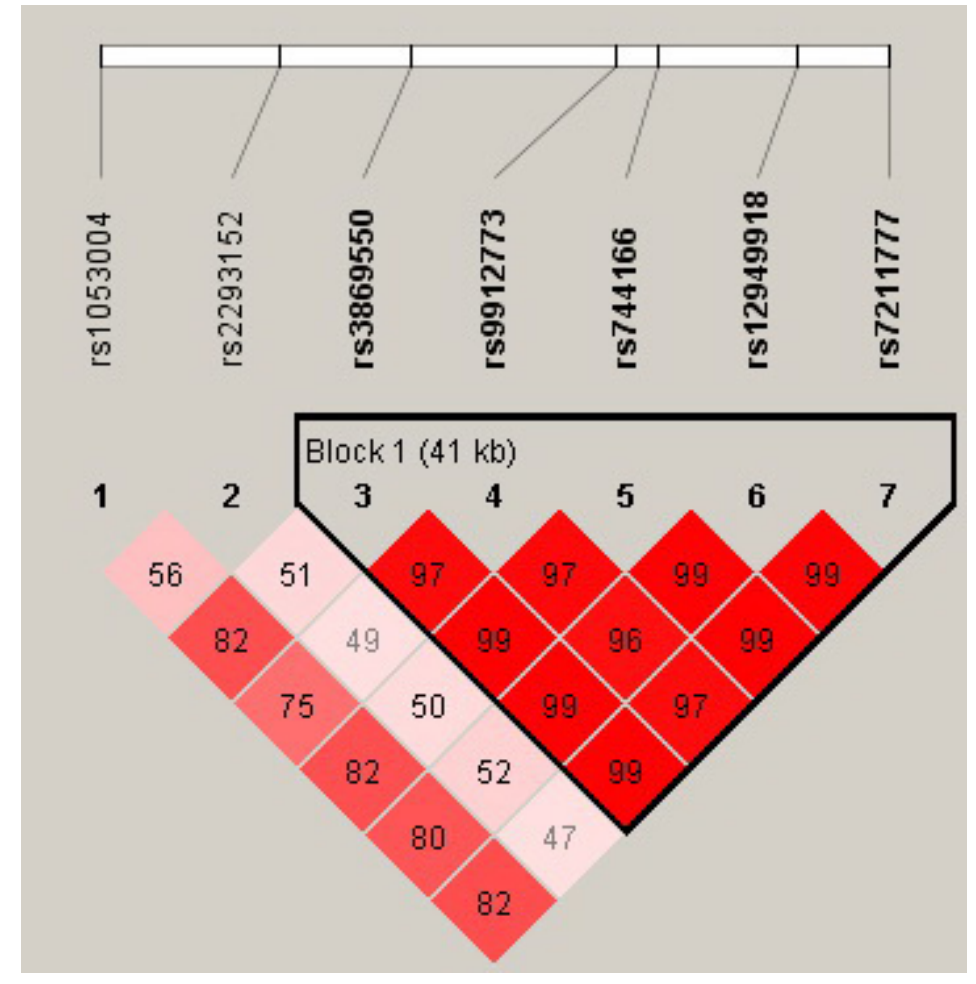

Figure 1. The linkage disequilibrium (LD) block structure observed in the STAT3 gene using the genotype data from the control sample. Each square box indicates the pairwise magnitudes of LD.

Stratification analyses by clinical stage showed that the trend for the association between STAT3 polymorphisms and NSCLC risk was present both for stage I/II and stage III/IV, and appeared moderately stronger for stage III/IV (Table 3). The ORs for stage III/IV NSCLC were $0.66(95 \% \mathrm{CI}=0.46-0.94), 0.65(95 \% \mathrm{CI}=0.46-0.93), 0.67(95 \% \mathrm{CI}=0.47-0.96), 0.68$ $(95 \% \mathrm{CI}=0.48-0.97), 0.68(95 \% \mathrm{CI}=0.48-0.98)$, and $0.67(95 \% \mathrm{CI}=0.47-0.95)$ for carriers of minor allele of rs4796793, rs7211777, rs12949918, rs744166, rs9912773, and rs3869550, respectively. Similarly, the minor allele haplotype of GGCGGC had a 0.72 -fold $(95 \% \mathrm{CI}=0.55$ 0.94 ) decreased risk of stage III/IV NSCLC. No significant associations of individual SNPs or haplotypes with NSCLC were observed when stratified by age, gender, cigarette smoking, and histology type (data not shown). 


\begin{tabular}{|c|c|c|c|c|c|}
\hline \multirow[t]{3}{*}{ SNP } & \multirow[t]{3}{*}{ Controls N (\%) } & \multicolumn{4}{|c|}{ Cases } \\
\hline & & \multicolumn{2}{|c|}{ Clinical stage I/II } & \multicolumn{2}{|c|}{ Clinical stage III/IV } \\
\hline & & $\mathrm{N}(\%)$ & OR $(95 \% \mathrm{CI})^{\dagger}$ & $\mathrm{N}(\%)$ & OR $(95 \% \mathrm{CI})^{\dagger}$ \\
\hline \multicolumn{6}{|l|}{ rs4796793 } \\
\hline $\mathrm{CC}$ & $159(36.9)$ & $65(45.1)$ & 1.00 (Reference) & $85(46.7)$ & 1.00 (Reference) \\
\hline CG or GG & $272(63.1)$ & $79(54.9)$ & $0.72(0.49-1.06)$ & $97(53.3)$ & $0.66(0.46-0.94)$ \\
\hline \multicolumn{6}{|l|}{ rs 7211777} \\
\hline AA & $158(36.7)$ & $66(45.8)$ & 1.00 (Reference) & $85(46.7)$ & 1.00 (Reference) \\
\hline $\mathrm{AG}$ or $\mathrm{GG}$ & $273(63.3)$ & $78(54.2)$ & $0.70(0.47-1.02)$ & $97(53.3)$ & $0.65(0.46-0.93)$ \\
\hline \multicolumn{6}{|l|}{ rs12949918 } \\
\hline TT & $161(37.5)$ & $64(44.8)$ & 1.00 (Reference) & $83(45.6)$ & 1.00 (Reference) \\
\hline $\mathrm{TC}$ or $\mathrm{CC}$ & $268(62.5)$ & $79(55.2)$ & $0.75(0.51-1.11)$ & $99(54.4)$ & $0.67(0.47-0.96)$ \\
\hline \multicolumn{6}{|l|}{ rs744166 } \\
\hline AA & $160(37.0)$ & $66(45.8)$ & 1.00 (Reference) & $84(46.2)$ & 1.00 (Reference) \\
\hline $\mathrm{AG}$ or $\mathrm{GG}$ & $272(63.0)$ & $78(54.2)$ & $0.71(0.48-1.04)$ & $98(53.9)$ & $0.68(0.48-0.97)$ \\
\hline \multicolumn{6}{|l|}{ rs9912773 } \\
\hline $\mathrm{CC}$ & $172(39.8)$ & $65(45.1)$ & 1.00 (Reference) & $87(47.8)$ & 1.00 (Reference) \\
\hline CG or GG & $260(60.2)$ & $79(54.9)$ & $0.82(0.56-1.20)$ & $95(52.2)$ & $0.68(0.48-0.98)$ \\
\hline \multicolumn{6}{|l|}{ rs3869550 } \\
\hline TT & $161(37.3)$ & $64(44.4)$ & 1.00 (Reference) & $85(46.7)$ & 1.00 (Reference) \\
\hline $\mathrm{TC}$ or $\mathrm{CC}$ & $271(62.7)$ & $80(55.6)$ & $0.76(0.51-1.11)$ & $97(53.3)$ & $0.67(0.47-0.95)$ \\
\hline \multicolumn{6}{|l|}{ Haplotype } \\
\hline CATACT & $517(59.8)$ & $182(63.3)$ & 1.00 (Reference) & $242(66.5)$ & 1.00 (Reference) \\
\hline GGCGGC & $312(36.2)$ & $94(32.6)$ & $0.86(0.64-1.14)$ & $105(28.9)$ & $0.72(0.55-0.94)$ \\
\hline GGCAGC & $15(1.7)$ & $3(1.0)$ & $0.62(0.18-2.17)$ & $8(2.1)$ & $1.07(0.44-2.57)$ \\
\hline Other haplotypes $\S$ & $20(2.3)$ & $9(3.1)$ & $1.18(0.52-2.65)$ & $9(2.5)$ & $1.00(0.45-2.23)$ \\
\hline
\end{tabular}

\section{DISCUSSION}

STAT3 transcriptional factor has been found to play pivotal roles in various aspects of tumor development and progression in a number of malignancies (Darnell, 2005; Fletcher et al., 2009; Yu et al., 2009). However, there is only one article that has investigated STAT3 polymorphisms in relation to cancer risk (Vaclavicek et al., 2007). Vaclavicek et al. (2007) reported that, although rs2293152 and rs7211777 in the STAT3 gene were not individually associated with familial breast cancer, the haplotypes characterized by rs 7211777 and a neighboring SNP rs6503691 in the STAT5B gene were found to modify the risk of familial breast cancer. In the present study, we showed for the first time that SNPs in the STAT3 gene were associated with decreased NSCLC risk. In particular, carriers of the minor alleles at SNP rs4796793, rs7211777, rs12949918, rs744166, rs9912773, or rs3869550 had a decreased risk of NSCLC, with ORs ranging from 0.67 to 0.75 . The GGCGGC haplotype comprised of all minor alleles at rs4796793, rs7211777, rs12949918, rs744166, rs9912773, and rs3869550 conferred a decreased risk of NSCLC with an OR of 0.78, supporting our findings based on individual SNPs. In a study of Crohn's disease, a significant association was observed between the rs744166 minor allele and decreased risk for Crohn's disease (Ferguson et al., 2010). Given that Crohn's disease has been recognized to have an increased risk of developing colorectal cancer, it is likely that rs 744166 minor allele is a protective factor for colorectal cancer, which is consistent with the findings on NSCLC in the present study.

Six STAT3 SNPs (rs4796793, rs7211777, rs12949918, rs744166, rs9912773, and 
rs3869550) and the haplotype characterized by them were found to modify the risk of NSCLC in the present study. Due to the strong LD (D' $\geq 0.96, r^{2} \geq 0.88$ ), it was impossible to identify a single causative SNP among the six. The exact underlying mechanism(s) responsible for our findings that STAT3 polymorphisms conferred decreased NSCLC risk remains unclear. All these SNPs are located in the $5^{\prime}$ region upstream and the introns of the STAT3 gene, suggesting that they may modify NSCLC risk by affecting gene transcriptional regulation and mRNA splicing. In vitro studies have shown that the genotypes of the SNP rs4796793 affected STAT3 mRNA levels, with the minor allele having a lower STAT3 expression (Ito et al., 2007). In the present study, rs4796793 was in strong LD (D' $\geq 0.97, r^{2} \geq 0.95$ ) with the other five NSCLCassociated SNPs (rs7211777, rs12949918, rs744166, rs9912773, and rs3869550). Thus, the STAT3 haplotype containing the minor alleles of the six SNPs may also correlate with lower STAT3 expression, and thus exert a protective effect on cancer development and progression. In line with this hypothesis, we observed that the six STAT3 SNPs and the haplotype characterized by them had a decreased risk for NSCLC, particular for stage III/IV NSCLC.

Strengths of this study include a relatively large sample size, good reproducibility of the genotyping results and careful study design of frequency-matching controls to NSCLC cases by age, gender and cigarette smoking. Limitations in our study should be noted. Design of hospital-based case-control studies may result in selection bias of participants, especially for controls who were cancer-free subjects from the same hospitals as cases. However, considering the high similarity in genotype and allele frequency between our controls and that in the HapMap Chinese database, it is reasonable to assume that the controls of our study represent the general Chinese population. Additionally, although up to 8 SNPs across the STAT3 gene were selected in the present study, the inclusion of SNPs was still limited and the SNPs examined may be in LD with untested genetic polymorphisms contributing to the results.

In summary, the STAT3 SNPs rs4796793, rs7211777, rs12949918, rs744166, rs9912773, and rs3869550, and the haplotype characterized by them were associated with decreased NSCLC risk in a Chinese population. Our data provided the first evidence that polymorphisms in the STAT3 gene may have a protective role in the development of NSCLC, particular of stage III/IV NSCLC. Because STAT3 polymorphisms have been correlated with therapy response in certain malignancies (Ito et al., 2007; Kreil et al., 2010), the STAT3 polymorphisms identified to be associated with decreased NSCLC risk in the present study could be helpful to predict how these patients would respond to therapy targeting STAT3 pathways. Future studies are needed to validate our findings and to investigate the potential effects of these SNPs on STAT3 expression and function.

\section{ACKNOWLEDGMENTS}

Research supported by the Shanghai Education Committee Foundation Grants (\#08YZ47); National Natural Science Foundation of China (\#30830038, \#30970842); Key Project of Science and Technology Commission of Shanghai Municipality (\#08JC1415000, \#08410702000, \#10JC1410000), and Shanghai Leading Academic Discipline Project (\#S30203).

\section{REFERENCES}

Abdulghani J, Gu L, Dagvadorj A, Lutz J, et al. (2008). Stat3 promotes metastatic progression of prostate cancer. Am. J. Pathol. 172: 1717-1728. 
Barrett JC, Fry B, Maller J and Daly MJ (2005). Haploview: analysis and visualization of LD and haplotype maps. Bioinformatics 21: 263-265.

Barrett JC, Hansoul S, Nicolae DL, Cho JH, et al. (2008). Genome-wide association defines more than 30 distinct susceptibility loci for Crohn's disease. Nat. Genet. 40: 955-962.

Bollrath J, Phesse TJ, von Burstin VA, Putoczki T, et al. (2009). gp130-mediated Stat3 activation in enterocytes regulates cell survival and cell-cycle progression during colitis-associated tumorigenesis. Cancer Cell 15: 91-102.

Bowman T, Garcia R, Turkson J and Jove R (2000). STATs in oncogenesis. Oncogene 19: 2474-2488.

Bromberg JF, Wrzeszczynska MH, Devgan G, Zhao Y, et al. (1999). Stat3 as an oncogene. Cell 98: 295-303.

Darnell JE (2005). Validating Stat3 in cancer therapy. Nat. Med. 11: 595-596.

Dauer DJ, Ferraro B, Song L, Yu B, et al. (2005). Stat3 regulates genes common to both wound healing and cancer. Oncogene 24: 3397-3408.

Ferguson LR, Han DY, Fraser AG, Huebner C, et al. (2010). Genetic factors in chronic inflammation: single nucleotide polymorphisms in the STAT-JAK pathway, susceptibility to DNA damage and Crohn's disease in a New Zealand population. Mutat. Res. 690: 108-115.

Fletcher S, Drewry JA, Shahani VM, Page BD, et al. (2009). Molecular disruption of oncogenic signal transducer and activator of transcription 3 (STAT3) protein. Biochem. Cell Biol. 87: 825-833.

Grivennikov SI and Karin M (2010). Dangerous liaisons: STAT3 and NF-kappaB collaboration and crosstalk in cancer. Cytokine Growth Factor Rev. 21: 11-19.

Horinaga M, Okita H, Nakashima J, Kanao K, et al. (2005). Clinical and pathologic significance of activation of signal transducer and activator of transcription 3 in prostate cancer. Urology 66: 671-675.

Hsieh FC, Cheng G and Lin J (2005). Evaluation of potential Stat3-regulated genes in human breast cancer. Biochem. Biophys. Res. Commun. 335: 292-299.

Hsu HS, Huang PI, Chang YL, Tzao C, et al. (2011). Cucurbitacin i inhibits tumorigenic ability and enhances radiochemosensitivity in nonsmall cell lung cancer-derived CD133-positive cells. Cancer 117: 2970-2985.

Ito N, Eto M, Nakamura E, Takahashi A, et al. (2007). STAT3 polymorphism predicts interferon-alfa response in patients with metastatic renal cell carcinoma. J. Clin. Oncol. 25: 2785-2791.

Jing N, Zhu Q, Yuan P, Li Y, et al. (2006). Targeting signal transducer and activator of transcription 3 with G-quartet oligonucleotides: a potential novel therapy for head and neck cancer. Mol. Cancer Ther. 5: 279-286.

Karin M (2006). Nuclear factor-kappaB in cancer development and progression. Nature 441: 431-436.

Kreil S, Waghorn K, Ernst T, Chase A, et al. (2010). A polymorphism associated with STAT3 expression and response of chronic myeloid leukemia to interferon alpha. Haematologica 95: 148-152.

Lai SY and Johnson FM (2010). Defining the role of the JAK-STAT pathway in head and neck and thoracic malignancies: implications for future therapeutic approaches. Drug Resist. Updat. 13: 67-78.

Lin Q, Lai R, Chirieac LR, Li C, et al. (2005). Constitutive activation of JAK3/STAT3 in colon carcinoma tumors and cell lines: inhibition of JAK3/STAT3 signaling induces apoptosis and cell cycle arrest of colon carcinoma cells. Am. J. Pathol. 167: 969-980.

Parkin DM, Bray F, Ferlay J and Pisani P (2005). Global cancer statistics, 2002. CA Cancer J. Clin. 55: 74-108.

Sansone P, Storci G, Tavolari S, Guarnieri T, et al. (2007). IL-6 triggers malignant features in mammospheres from human ductal breast carcinoma and normal mammary gland. J. Clin. Invest. 117: 3988-4002.

Scagliotti GV, Longo M and Novello S (2009). Nonsmall cell lung cancer in never smokers. Curr. Opin. Oncol. 21: 99-104.

Shields PG (2002). Molecular epidemiology of smoking and lung cancer. Oncogene 21: 6870-6876.

Siddiquee K, Zhang S, Guida WC, Blaskovich MA, et al. (2007a). Selective chemical probe inhibitor of Stat3, identified through structure-based virtual screening, induces antitumor activity. Proc. Natl. Acad. Sci. U. S. A. 104: 7391-7396.

Siddiquee KA, Gunning PT, Glenn M, Katt WP, et al. (2007b). An oxazole-based small-molecule Stat3 inhibitor modulates Stat3 stability and processing and induces antitumor cell effects. ACS Chem. Biol. 2: 787-798.

Stephens M, Smith NJ and Donnelly P (2001). A new statistical method for haplotype reconstruction from population data. Am. J. Hum. Genet. 68: 978-989.

Turkson J (2004). STAT proteins as novel targets for cancer drug discovery. Expert. Opin. Ther. Targets. 8: 409-422.

Vaclavicek A, Bermejo JL, Schmutzler RK, Sutter C, et al. (2007). Polymorphisms in the Janus kinase 2 (JAK)/signal transducer and activator of transcription (STAT) genes: putative association of the STAT gene region with familial breast cancer. Endocr. Relat. Cancer 14: 267-277.

Weerasinghe P, Garcia GE, Zhu Q, Yuan P, et al. (2007). Inhibition of Stat3 activation and tumor growth suppression of non-small cell lung cancer by G-quartet oligonucleotides. Int. J. Oncol. 31: 129-136.

Yang L, Parkin DM, Li LD, Chen YD, et al. (2004). Estimation and projection of the national profile of cancer mortality in China: 1991-2005. Br. J. Cancer 90: 2157-2166.

Yin W, Cheepala S, Roberts JN, Syson-Chan K, et al. (2006). Active Stat3 is required for survival of human squamous cell carcinoma cells in serum-free conditions. Mol. Cancer 5: 15. 
Yin ZJ, Jin FG, Liu TG, Fu EQ, et al. (2010). Overexpression of STAT3 potentiates growth, survival, and radioresistance of non-small-cell lung cancer (NSCLC) cells. J. Surg. Res. Doi: 10.1016/j.jss.2010.03.053.

Yu H and Jove R (2004). The STATs of cancer - new molecular targets come of age. Nat. Rev. Cancer 4: 97-105.

Yu H, Pardoll D and Jove R (2009). STATs in cancer inflammation and immunity: a leading role for STAT3. Nat. Rev. Cancer 9: 798-809. 